\title{
Validation d'une procédure de prise en charge des mucites induites par les inhibiteurs de la voir mTOR en oncologie : proposition d'une étude prospective.
}

\author{
Calvo AS ${ }^{1,2}$, Malouf $\mathrm{G}^{3}$, Descroix $\mathbf{V}^{1,2}$, Pierga JY4, Lescaille G1,2,5 \\ 1. Service d'Odontologie, Groupe Hospitalier Pitié-Salpêtrière - Assistance Publique-Hôpitaux de Paris (AP-HP) - Paris \\ 2. Université Paris Diderot/Paris 07 , Sorbonne Paris Cite \\ 3. Service d'Oncologie Médicale, Groupe Hospitalier Pitié-Salpêtrière - Assistance Publique-Hôpitaux de Paris (AP-HP) - Paris \\ 4. Service d'Oncologie Médicale, Institut Curie, Paris \\ 5. Centre d'Immunologie et Maladies Infectieuses (CIMI-Paris), UMR-S INSERM U1135, CNRS ERL 8255, Sorbonne Universi- \\ tés, UPMC/Paris 06
}

L'évérolimus est un agent antinéoplasique, inhibiteur sélectif de la voie mTOR impliquée dans les le processus tumoral de nombreux cancers. Ses indications oncologiques sont : les cancers rénaux, certaines tumeurs neuroendocrines et les cancers du sein. Les lésions buccales de type aphtoïdes sont fréquentes au cours de ces traitements, autour de $40 \%$ après 15 jours. Si le laser de faible puissance a montré de bons résultats sur les mucites associées aux traitements de chimiothérapie/radiothérapie, il n'y a pas de preuve scientifique établissant une efficacité sur ces lésions spécifiques. En revanche, les bains de bouche de corticoïdes permettraient de diminuer l'incidence des mucites induites par l'everolimus (ref asco). II s'agira d'une étude prospective dans le cadre d'une collaboration avec les services d'oncologie de la Pitié Salpétrière et de l'institut Curie, dont l'objectif principal est de valider l'efficacité d'un protocole de prise en charge des mucites buccales induites par l'everolimus ; il s'agit de traiter les mucites dès apparition d'un stade 1 par des bains de bouche de corticoïde (methyl prednisolone). En cas d'évolution vers un stade 2, un traitement par laser de faible puissance (diode) à raison de 2 fois/semaine sera proposé jusqu'à retour à un stade 1. Les objectifs secondaires seront de mieux caractériser ces lésions d'un point de vue clinique (caractérisation des lésions et échelle NCl-CTCAE 3.0, répercussions sur la qualité de vie et questionnaire QLQHN35) et de tenter de mettre en évidence d'éventuels facteurs prédisposants au développement des lésions ou de réponse au traitement proposé. Tous les patients auront une consultation bucco-dentaire et une remise en état bucco -dentaire avant de débuter le traitement oncologique avec un prélèvement salivaire et collecte des données odontologiques (statut parodontal et carieux, caractérisation salivaire). Un recueil d'un échantillon salivaire sera également réalisé à $15 \mathrm{j}$ après le début du traitement, puis avant chaque séance de laser de faible puissance. Cette étude nécessitera le recueil d'un consentement éclairé ainsi qu'un avis favorable du comité d'éthique.

Les résultats attendus sont une diminution de prévalence des mucites sévères. L'analyse des échantillons salivaires permettra par la suite de proposer une étude de caractérisation du caractère inflammatoire de ces mucites, mais également de potentiellement identifier des profils à risque ou encore de monitorer la réponse aux traitements locaux proposés.

Les mucites induites par inhibiteurs de mTor sont importantes à prendre en compte car elles entrainent une réduction des doses dans $8,2 \%$ dans cas voir même un arrêt du traitement chez plus de $12 \%$ des patients. L'évérolimus a un mode d'administration oral au domicile et l'arrêt du traitement se fait souvent du propre chef du patient. Une meilleure prise en charge de ces lésions par l'élaboration d'un protocole précis est nécessaire.

a.sophiecalvo@gmail.com

Références

Rugo H et al: International Symposium on Supportive Care in Cancer. Abstract MASCC-0638. Presented June 23, 2016.

Peterson DE et al. Cancer Med. 2016 Aug;5(8):1897-1907

Niikura N et al. J Clin Oncol. 2016 Sep;46(9):879-882

(C) The authors, published by EDP Sciences. This is an Open Access article distributed under the terms of the Creative Commons Attribution License 4.0 (http://creativecommons.org/licenses/by/4.0/). 